Йзв. АН Эстонии, Физ. Матем., 1989, 38, № 3, 288-293

удК 537.632

Г. ЛиИдья, Т. РЫЫМ

\title{
СПИН-РЕШЕТОЧНАЯ РЕЛАКСАЦИЯ F+-ЩЕНТРОВ В КРИСТАЛЛЕ СаО
}

Время спин-решеточной релаксации в облученном нейтрянами монокристалле $\mathrm{CaO}$ измерено по магнитному циркулярному дихроизму в $\mathrm{F}^{+}$-полосе поглощения. В интервале магнитных полей от $10^{-2}$ до 3 Тл обнаружена аномальная зависимость скорости релаксации от магнитного поля, близкая к $\tau^{-1} \sim H^{-1}$ при $T=2 \mathrm{~K}$, и температуры $\tau^{-1} \sim \mathrm{T} \alpha$, где $\alpha=1,8 \pm 0,2$. Релаксация такого типа связана, вероятно, с примесями. Удовлетворительного теоретического объяснения обнаруженной полевой зависимости нет.

\section{1. Введение}

Тепловое равновесие в кристаллах с парамагнитными примесями устанавливается путем обмена энергии между спиновой и фононной подсистемами. Если концентрация парамагнитных дефектов мала (спины изолированы) и они все одного сорта, то развиваются т. н. собственные процессы спин-фононной релаксации. При низких температурах преобладают прямые процессы, в которых переход между двумя спиновыми подуровнями сопровождается поглощением или испусканием одного фонона. Такие процессы хорошо изучены в F-центрах щелочно-галоидных кристаллов (один электрон в анионной вакансии): $\left.\mathrm{KCl}\left[{ }^{1}\right], \mathrm{KI}, \mathrm{KBr}\left[{ }^{2}\right], \mathrm{NaCl}_{[3}^{3}\right]$. Установлено, что скорость (обратное время) релаксации

$$
\tau_{1}^{-1}=\left(A H^{3}+B H^{5}\right) \operatorname{coth}\left(g \mu_{B} H / 2 k T\right),
$$

ғде $H$ - индукция магнитного поля, $\mu_{B}-$ магнетон Бора, $k-$ постоянная Больцмана, $T-$ абсолютная температура, $g \approx 2-$ электронный $g$-фактор. Формула (1) верна в пределе низких частот дебаевского приближения, когда закон дисперсии фононов можно считать линейным. Первый член $\sim H^{3}$ обусловлен модуляцией сверхтонкого взаимодействия колебаниями решетки $\left[{ }^{4}\right]$. С повышением магнитного поля скорость релаксации растет как плотность энергии фононного поля на частоте перехода $\mathrm{Q}_{\varepsilon} \sim(\hbar \omega)^{3}=\left(g \mu_{B} H\right)^{3}$; Второй член в (1) обусловлен модуляцией электроєтатического потенциала электрона $\left[{ }^{5}\right]$. Особенностью парамагнитных центров, где основное состояние есть Крамерсов дублет (к ним относя́тся центры окраски с вырожденным при $H=0$ основным состоянием), является пятая степень зависимости скорости релаксации от поля. Дополнительный множитель $\sim H^{2}$ происходит от примеси возбужденного состояния, орбитальный момент которого непосредственно модулируется колебаниями.

В простых окислах двухвалентных металлов один электрон в вакансии кислорода принадлежит т. н. $\mathrm{F}^{+}$-центру. Особенный интерес представляет СaO. Естественный изотопный состав кальция $\left(0,13 \%{ }^{43} \mathrm{Ca}\right.$; остальные $99,87 \%$ ядер имеют нулевой спин) и кислорода $\left(0,04 \%{ }^{17} \mathrm{O}\right.$; остальные $99,96 \%$ ядер имеют нулевой спин) таков, что лишь один из $40 \mathrm{~F}^{+}$-центров имеет в первых трех конфигурационных сферах отличный от нуля спин ядра. Следовательно, в кристалле $\mathrm{CaO}$ релаксация спина $\mathrm{F}^{+}$-центра путем модуляции сверхтонкого взаимодействия практически не осуществляется и в чистом от примесей кристалле должен работать лишь механизм Кронига-Ван Флека $\left(\sim H^{5}\right)$. Собственная релаксация в слабых полях должна быть исключительно медленной 
(см. раздел 4). В результате открывается широкая область магнитных полей, в которой могут отчетливо проявляться несобственные механизмы релаксации.

Время спин-решеточной релаксации $\mathrm{F}^{+}$-центров в $\mathrm{CaO}$ измерялось в $\left[{ }^{6}\right]$. При $T=1,7 \mathrm{~K}$ и $H=1,1$ Тл время колебалось от 5 до 25 с в зависимости от термической обработки кристаллов. Примерно такие же времена $\left(4-10\right.$ с) измерены для $\mathrm{F}_{A^{+}}$-центров, имеющих в первой конфигурационной сфере примесный ион магния $\left[{ }^{7}\right]$ или лития $\left[{ }^{8}\right]$. O полевой зависимости $\tau$ в литературе данные отсутствуют.

\section{2. Объекты и методика измерения}

Бесцветные прозрачные монокристаллы $\mathrm{CaO}$ (99,95\% чистоты), полученные от английской фирмы «W. C. Spicer Ltd», подвергались облучению нейтронами $\left(1,7 \cdot 10^{17} \mathrm{~cm}^{-2}\right.$ быстрых нейтронов в нефильтрованном потоке). Перед проведением оптических измерений приповерхностный слой кристалла удаляли шлифованием, затем большие поверхности полировали. Концентрация $\mathrm{F}^{+}$-центров, оцененная по оптическому поглощению, была $7 \cdot 10^{16} \mathrm{~cm}^{-3}$. Перед измерением спиновой релаксации кристалл подвергали термической обработке, в результате которой концентрация $\mathrm{F}^{+}$-центров уменьшалась до $3 \cdot 10^{16} \mathrm{~cm}^{-3}$.

Магнитный циркулярный дихроизм измеряли на дихрографе ДХР-02, приспособленном для магнитооптических измерений. С этой целью термостатируемая камера объекта была заменена на гелиевый криостат с кварцевыми окнами, содержащий сверхпроводящий магнит с горизонтальной осью (длина соленоида 40 мм, внутренний диаметр 14 мм, постоянная по току 60 мТл. $A^{-1}$ ). Объект (пластинка размерами $8 \times 7 \times$ $\chi 1$ м $\left.^{3}\right)$ в оправе помещали в центр соленоида, где его температура могла быть понижена путем откачки паров гелия ниже $\lambda$-точки $(2,17 \mathrm{~K})$.

На рис. 1 представлена спектральная зависимость сигнала дихроизма $\mathrm{CaO}$ в поле $H=2,38$ Тл. Спектр измерен через широкие щели $(\Delta \lambda=13 \AA)$, поэтому в нем преобладает широкая электронноколебательная полоса $\mathrm{F}^{+}$-центра, а особенность, связанная с узкой бесфононной линией при $\lambda=$ $=3357 \AA$ А, выражена слабо (см. также $\left.\left[{ }^{9}\right]\right)$.

Сигнал дихроизма $\Delta k_{ \pm}$(раз-

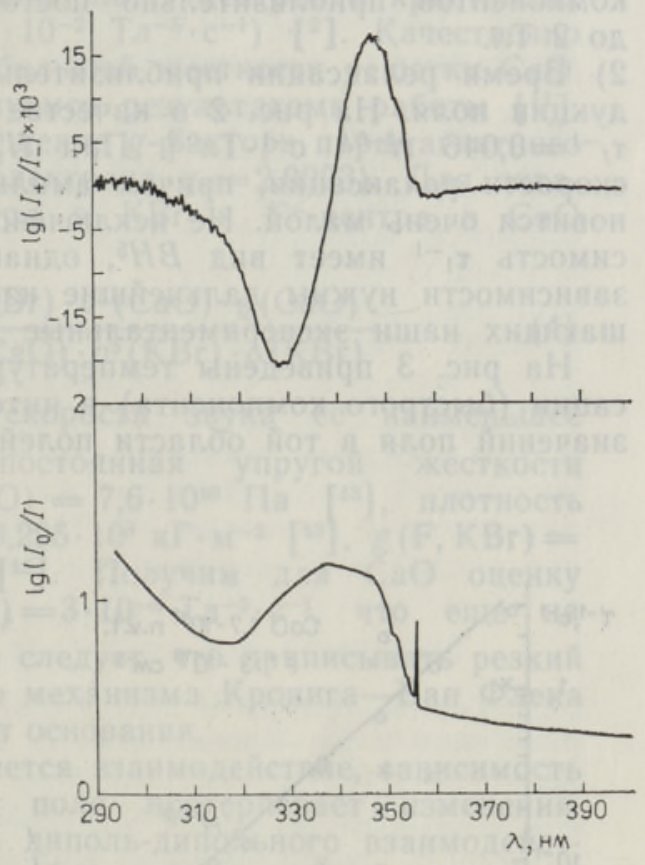

Рис. 1. Спектральная зависимость кругового дихроизма $(T=2,14 \quad \mathrm{~K}, \quad H=$ $=2,38$ Тл) и спектр поглощения ( $T=$ $=4,2 \mathrm{~K})$ кристалла $\mathrm{CaO}$, облученного в реакторе $\left(n_{F+} \approx 3 \cdot 10^{1 \tilde{}} \mathrm{cm}^{-3}\right)$ пость поглощения право- и левополяризованного света) в центрах окраски обусловлен в основном относительным смещением электронноколебательных полос $\Delta E_{ \pm}$, которое в свою очередь включает диамагнитную и парамагнитную часть $\left[{ }^{10}\right]$.

$$
D \sim \Delta k_{ \pm} \sim \Delta E_{ \pm}= \pm\left[A_{\mathrm{dia}} \cdot H+A_{\mathrm{para}} \cdot\left\langle S_{Z}\right\rangle\right] .
$$

Первый член следует магнитному полю адиабатически. Второй член 
пропорционален мгновенному значению среднего спинового момента в направлении распространения света.

В измерениях времени спин-решеточной релаксации $\tau(H)$ сигнал дихроизма на длине волны $\lambda=3470 \AA$ регистрировался при скачкообразном изменении магнитного поля со скоростью $d H / d t \approx 1$ Тл $\mathrm{c}^{-1}$ и амплитудой $\sim 1$ Тл. Временной ход сигнала с усилителя дихрографа, имеющего постоянную времени около 0,2 с, записывался в память ӘВМ «Aspect 3000». Парамагнитный (инерционный) вклад в сигнал дихроизма сильно превышает диамагнитный при $\lambda=3470 \AA ̊$. Аппроксимация временной зависимости с помощью суммы двух экспонентов осуществлялась методом наименьших квадратов.

\section{3. Результаты}

На рис. 2 изображена зависимость скорости релаксации $\tau_{1}^{-1}$ от индукции магнитного поля. В магнитных полях $H<3$ Тл наблюдаются следующие закономерности : 1) Релаксация состоит из двух экспоненциальных компонентов, причем быстрый компонент включает в себя около $80 \%$ начальной амплитуды и имеет постоянную $\tau_{f}$ в 4 раза короче медленного компонента $\tau_{s}$. Соотношения амплитуд и времен компонентов приблизительно постоянны во всем интервале от 0,01 до 2 Тл.

2) Время релаксации приблизительно пропорционально величине индукции поля. На рис. 2 в качестве аппроксимации приведена прямая $\tau_{f}^{-1}=0,046 H^{-0,9} \mathrm{c}^{-1} \cdot \mathrm{T}^{0,9}$. При $H \geqslant 3$ Тл наблюдается резкий рост скорости релаксации, причем амплитуда медленного компонента становится очень малой. Не исключено, что при $H>3$ Тл полевая зависимость $\tau_{1}^{-1}$ имеет вид $B H^{5}$, однако для точного определения этой зависимости нужны дальнейшие измерения в сильных полях, превышающих наши экспериментальные возможности (см. раздел 4).

$\mathrm{Ha}$ рис. 3 приведены температурные зависимости скорости релаксации (быстрого компонента) в интервале $2,14-4,21 \mathrm{~K}$ для некоторых значений поля в той области полей, где $\tau_{1}$ возрастает с напряженно-

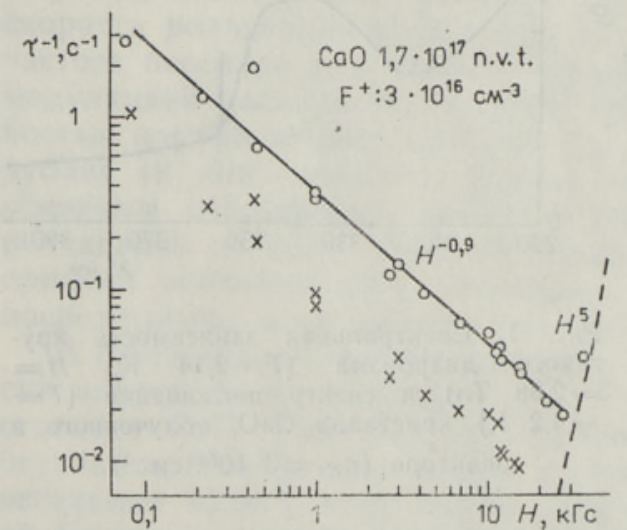

Рис. 2. Зависимость скорости спин-решеточной релаксации $\mathrm{F}^{+}$-центров $\mathrm{CaO}$ от магнитного поля при $T=2,14 \mathrm{~K}$. Точки - быстрый, крестики - медленный компонент двухэкспоненциального разложения.

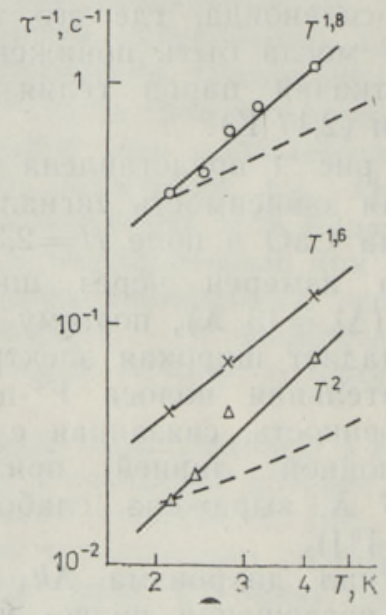

Рис. 3. Зависимогть скорости спин-решеточной релаксащии $\mathrm{F}^{+}$-центров $\mathrm{CaO}$ от температуры в голе 0,1 Тл (кружки), 1,2 Тл (крестики) и 2,65 Тл (треугольники). Показаны аппроксимации степенным законом $T \alpha$ и однофононным приближением $\operatorname{coth}(g \mu H / 2 k T)$ (пунктир). 
стью поля. В узком интервале температурная зависимость может быть аппроксимирована степенной зависимостью

$$
\tau_{1}^{-1} \sim T^{1,8 \pm 0,2} .
$$

Характерная для прямого спин-фононного механизма релаксации зависимость $\tau_{1}^{-1} \sim \operatorname{coth}\left(g \mu_{B} H / 2 k T\right)$ в указанном интервале магнитных полей не соответствует эксперименту.

Таким образом, для широкой области магнитных полей (от 0,01 до 3 Тл) установлена аномальная зависимость скорости спин-решеточной релаксации $\mathrm{F}^{+}$-центров $\mathrm{CaO}$ от магнитного поля и температуры.

\section{4. Обсуждение результатов}

Найденная нами при $T=2,14 \mathrm{~K}$ зависимость $\tau_{1}^{-1}(H)$ может быть подогнана суммой

$$
\tau_{1}^{-1}=B_{e} H^{5} \operatorname{coth}\left(g \mu_{B} H / 2 k T\right)+0,046 H^{-0,9},
$$

где $B_{e}=(3,5 \pm 1) \cdot 10^{-5}$ Тл ${ }^{-5} \cdot \mathrm{c}^{-1}$. Рассмотрим, может ли быстрый рост $\tau_{1}^{-1}$ при $H>3$ Тл быть обусловлен механизмом Кронига-Ван Флека для изолированных $\mathrm{F}^{+}$-центров. Полученное значение $B_{e}$ на несколько порядков меньше аналогичной величины для $\mathrm{F}$-центров в кристаллах $\mathrm{KBr}\left(1,68 \cdot 10^{-3} \mathrm{~T}^{-5} \cdot \mathrm{c}^{-1}\right)$ или $\left.\mathrm{KI}\left(1 \cdot 10^{-2} \mathrm{~T}^{-5} \cdot \mathrm{c}^{-1}\right){ }^{2}\right]$. Качественно это различие могло быть приписано большей жесткости решетки $\mathrm{CaO}$. Для количественной оценки воспользуемся результатами работы [ $\left.{ }^{11}\right]$, где получено выражение $B_{t}$ через смещение $g$-фактора парамагнитного центра $\Delta g=g-g_{e}$ (для свободного электрона $\left.g_{e}=2,0023\right)$. Для октаэдрически координированных $\mathrm{F}$-центра в $\mathrm{KBr}$ и $\mathrm{F}^{+}$-центра в $\mathrm{CaO}$ можно записать

$$
\frac{B_{t}(\mathrm{KBr})}{B_{t}(\mathrm{CaO})}=\frac{[\Delta g(\mathrm{KBr})]^{2} \cdot g^{3}(\mathrm{KBr}) \cdot v^{5}(\mathrm{CaO}) \cdot \mathrm{Q}(\mathrm{CaO})}{[\Delta g(\mathrm{CaO})]^{2} \cdot g^{3}(\mathrm{CaO}) \cdot v^{5}(\mathrm{KBr}) \cdot \mathrm{Q}(\mathrm{KBr})} .
$$

Для оценки возьмем в качестве скорости звука ее наименьшее значение $v_{t}\langle 100\rangle=\sqrt{C_{44} / \mathrm{Q}}$, где постоянная упругой жесткости $C_{44}(\mathrm{KBr})=5,04 \cdot 10^{9} \Pi^{4}\left[{ }^{12}\right], \quad C_{44}(\mathrm{CaO})=7,6 \cdot 10^{10} \Pi^{10}\left[{ }^{13}\right]$, плотность $\mathrm{\varrho}(\mathrm{KBr})=2,75 \cdot 10^{3}$ кГ $\mathrm{M}^{-3}$ и $\mathrm{\varrho}(\mathrm{CaO})=3,295 \cdot 10^{3} \mathrm{\kappa} \Gamma \cdot \mathrm{M}^{-3}\left[{ }^{13}\right], g(\mathrm{~F}, \mathrm{KBr})=$ $=1,982\left[{ }^{14}\right], g\left(\mathrm{~F}^{+}, \mathrm{CaO}\right)=2,0001 \quad\left[{ }^{15}\right]$. Получим для $\mathrm{CaO}$ оценку $B(\mathrm{CaO})=B(\mathrm{KBr}) \cdot B_{t}(\mathrm{CaO}) \cdot B_{t}^{-1}(\mathrm{KBr})=3 \cdot 10^{-8} \mathrm{~T}^{-5} \cdot \mathrm{c}^{-1}$, что еще на 3 порядка меньше $B_{e}(\mathrm{CaO})$. Отсюда следует, что приписывать резкий рост $\tau^{-1}(H)$ при $H>3$ Тл включению механизма Кронига-Ван Флека на изолированных $\mathrm{F}^{+}$-центрах не имеет основания.

Если между $\mathrm{F}^{+}$-центрами наблюдается взаимодействие, зависимость скорости релаксации от магнитного поля претерпевает изменения. Соответствующие поправки в случае диполь-дипольного взаимодействия рассмотрены в $\left[{ }^{16-18}\right]$, в случае обменного взаимодействия - в $\left[{ }^{18}\right]$. Взаимодействие между парамагнитными центрами может привести к возрастанию скорости релаксации в сторону малых полей. Такие эффекты проявляются в некоторых парамагнитных системах $[2,19,20]$ в полях, сравнимых с внутренними (доли тесла). Однако теории, позволяющей объяснить простирающую через десятки килогаусс зависимость типа $\tau_{1} \sim H$, как это имеет место в СаO, по-видимому, нет.

Возможно, что аномальная зависимость $\tau_{1}(H)$ связана с релаксацией $\mathrm{F}^{+}$-центров на примесях, в том числе на таких, которые имеют ненулевой спин ядра. В качестве таких примесей наряду с уже упомянутыми $\mathrm{Li}^{+}\left[{ }^{8}\right]$ и $\mathrm{Mg}^{2+}\left[{ }^{7}\right]$ может быть рассмотрен водород $\left(\mathrm{H}^{2-}\left[{ }^{21}\right], \mathrm{OH}^{2-}\left[{ }^{22}\right], V_{\mathrm{OH}^{-}}\left[{ }^{23}\right]\right)$.

Согласно $\left[{ }^{8}\right]$, к спадающей с полем скорости релаксации может 
привести примесь, совершающая туннельные переходы. При этом мо̄дулируется сверхтонкое взаимодействие $\hat{H}_{S F}=\vec{S} \cdot \hat{D} \cdot \vec{I} \quad$ между спином $\vec{S} \mathrm{~F}^{+}$-центра и ядерным моментом $\vec{I}$ туннелирующей примеси, которая непосредственно взаимодействует с фононами. Если туннельное расщепление $E$ больше зеемановского, то скорость спин-решеточной релаксации по указанному механизму

$$
\tau_{01}^{-1}=\frac{3}{4 \pi \hbar^{4} \varrho^{-5}} \frac{\left|\left\langle H_{S F}\right\rangle\right|^{2}}{E}\left(\frac{G_{+}^{2}}{E^{2}}+\frac{G_{-}^{2}}{\left(g \mu_{B} H\right)^{2}}\right) \cdot \operatorname{cosech}(E / k T),
$$

где $E=\sqrt{\Delta^{2}+\xi^{2}}, \quad \Delta-$ энергия перекрывания, $\xi-$ величина асимметрии потенцилльных ям локального туннельного состояния. Величины

$$
\begin{aligned}
& G_{+}^{2}=\Delta^{2}(\xi \partial \xi / \partial e+\Delta \partial \Delta / \partial e)^{2}, \\
& G_{-}^{2}=\xi^{2}(\xi \partial \Delta / \partial e-\Delta \partial \xi / \partial e)^{2}
\end{aligned}
$$

выражают величину связи туннелирующей системы с деформацией $e$. Согласно (5), в области слабых полей может наблюдаться зависнмость $\tau_{01}^{-1} \sim \mathrm{H}^{-2}$, переходящая в $\tau_{01}^{-1}=$ const в сильных полях. Температурная зависимость $\tau_{01}^{-1}$ имеет вид $\tau_{0,1}^{-1} \sim T$ при $T \gg E / k$ и $\tau_{01}^{-1} \sim \exp (-E / k T)$ при $T \ll E / k$. Все эти зависимости не соответствуют нашим экспериментальным результатам по СаО. Не исключено, однако, что включение в рассмотрение нескольких сортов туннелирующих примесей позволяет получить зависимости $\tau^{-1}(H)$ и $\tau^{-1}(T)$, более близкие к эксперименту.

Если концентрация $\mathrm{F}^{+}$-центров больше чем концентрация туннелирующих примесей, не все $\mathrm{F}^{+}$-центры могут иметь поблизости примеси. Тогда возможно, что релаксация описывается диффузией спинов по $\mathrm{F}^{+}$-центрам к быстро релаксирующим центрам типа $\mathrm{F}_{A^{+}}$и т. д., имеющим в соседстве туннелирующее ядро примеси. Формально такой процесс может быть описан так же, как и диффузия ядерных спинов к парамагнитным примесям $\left[{ }^{24,}{ }^{25}\right]$. В случае свободной диффузии поведение ансамбля $\mathrm{F}^{+}$-центров описывается скоростью релаксации

$$
T_{1}^{-1}\left(\mathrm{~F}^{+}\right)=4 \pi n_{\mathrm{F}_{A}^{+}} \cdot b \cdot D
$$

где $n_{\mathrm{F}_{A}^{*}}$ - концентрация центров быстрой релаксации, $b-$ длина рассеяния и $D-$ коэффициент диффузии. Зависимость $T_{1}^{-1}$ от $H$ появляется через длину рассеяния $\left[{ }^{24}\right]$, которая сокращается при возрастапии поля из-за неравенства $g$-факторов $\mathrm{F}_{A}^{+}$и $\mathrm{F}^{+}$-центров. Применимость диффузионного приближения вызывает, однако, сомнения, потому что средняя длина свободного пробега, равная среднему расстояни: между $\mathrm{F}^{+}$-центрами $n_{\mathrm{F}^{*}}{ }^{-1 / 3}$, весьма большая ( 100 постоянных решетки в нашем кристалле).

Таким образом, нами обнаружена спин-решеточная релаксация $\mathrm{F}^{+}$-центров в окрашенных быстрыми нейтронами кристаллах $\mathrm{CaO}$, полевая зависимость которой не описывается существующими теориями. Вероятнее всего, релаксация обусловлена примесями. СаO представляет собой уникальный объект исследования таких процессов, потому что процессы собственной релаксации вступают в сілу лишь в сильных полях. Недавно была найдена аналогичная полевгя зависимость скорости протонной спин-решеточной релаксации в этилсульфате иттрия $\left[{ }^{26}\right]$, не получившая теоретического объяснения. 
В заключение авторы выражают признательность К. Шварцу за предоставление возможности провести облучение на исследовательском реакторе Института физики АН Латвийской ССР в Саласпилсе и У. Нагелю за участие в измерениях.

\section{Л И Т Е Р А Т У Р А}

1. Feldman, D. W., Warren, R. W., Castle, J. G. Jr. // Phys. Rev., 1964, 135, A470.

2. Panepucci, H., Mollenauer, L. F. // Phys. Rev., 1969, 178, A589.

3. Carvalho, R. A., Panepucci, H., Terrile, M. C. // Phys. Rev., 1977, B15, 1116.

4. Дейген М. Ф., Зевин В. Я. // ЖЭТФ, 1960, 36, 1126.

5. Kronig, R. de L. // Physica, 1939, 6, 33; Van Vleck, J. H. // Phys. Rev., 1940, $57,426$.

6. Kemp, J. C., Ziniker, W. M., Glaze, J. A. // Proc. British Ceramic Soc., 1967, 9. 109 .

7. Ahlers, F. J., Lohse, F., Spaeth, J. M. // Solid State Commun., 1982, 43, 321.

8. Stinson, D. G., Stapleton, H. I. // Phys. Rev., 1983, B27, 5386.

9. Merle d'Aubigné, Y., Roussel, A. // Phys. Rev., 1971, B3, 1421.

10. Mort, J., Lüty, F., Brown, F. C. // Phys. Rev., 1965, 137, A566.

11. Terrile, M. C., Panepucci, H., Helman, J. S. // Nuovo cimento, 1979, 53B, 446.

12. Garg, V. K., Puri, D. S., Verma, M. P. // Phys. stat. sol. (b) 1977, 80, 63.

13. Hite, H. E., Kearney, R. J. // J. Appl. Phys., 1967, 38, 5424.

14. Seidel, H., Wolf, H. C. // Physics of Color Centers (ed. W. Beall Fowler). New York, London, Academic Press, 1968, 555.

15. Henderson, B., Tomlinson, A. C. // J. Phys. Chem. Sol., 1969, 30, 1801

16. Orbach, R. // Proc. Roy. Soc. London, 1961, A264, 485.

17. Huber, D. L. // Phys. Rev., 1963, 131, 190.

18. Глинчук М. Д., Грачев В. Г., Дейген М. Ф. // ФТТ, 1966, 8, 3354.

19. Warren, R. W., Feldman, D. W., Castle, J. G. Jr. // Phys. Rev., 1964, 136, A1347.

20. Moore, C. A. // Phys. Rev., 1976, B13, 1925.

21. Orera, V. M.. Chen, Y. // Phys. Rev.. 1987, B36. 1244.

22. Sanjuan, M. L., Orera, V. M. // J. Chem. Phys., 1986, 85, 4254.

23. Henderson, B., Wertz, J. E. Defects in the Alkaline Earth Oxides. London, Taylor \& Francis Ltd., 1977, 61.

24. Jeffries, C. D. Dynamic Nuclear Orientation. New York etc., Interscience Publ., 1963

25. Александров Н. В. Теория магнитной релаксации. М., Наука, 1975, 326.

26. Sowinski, J., Knutson, L. D. // Phys. Rev., 1988, B37, 9208.

Ннститут химической и биологической физики Академии наук Эстонской ССР
Поступила в редакцию 12/XII 1988

\section{G. LIIDJA, T. ROOM}

\section{$\mathrm{F}^{+}$-TSENTRITE SPINN-VORE RELAKSATSIOON $\mathrm{CaO}$ KRISTALLIS}

On mõõdetud neutronvoos värvitud $\mathrm{CaO}$ monokristalli $\mathrm{F}^{+}$-tsentrite paramagnetilist relaksatsiooni valguse $(347 \mathrm{~nm})$ tsirkulaarse dikroismi signaali aegsôltuvuse kaudu astmeliselt muutuvas magnetväljas temperatuuril $2-4 \mathrm{~K}$. Magnetvälja induktsioonivahemikus $10^{-2}-3 \mathrm{~T}$ on leitud relaksatsioonikiiruse anomaalne sõltuvus magnetväljast (ligikaudu $\left.\tau^{-1} \sim H^{-1}\right)$ ja temperatuurist $\left(\tau^{-1} \sim \mathrm{T}^{1,8 \pm 0,2}\right)$. Seni teadaolevad relaksatsiooniteooriad ei anna rahuldavat kvantitatiivset seletust leitud sōltuvustele.

\section{G. LIIDJA and T. ROOM}

\section{SPIN-LATTICE RELAXATION OF F+ CENTERS IN A CaO CRYSTAL}

Spin-lattice relaxation times have been measured for a neutron irradiated $\mathrm{CaO}$ monocrystal with $3 \cdot 10^{16} \mathrm{~F}^{+}$centers per cubic centimeter, using magnetic circular dicroism in the broad phonon sideband of $\mathrm{F}^{+}$centers. An anomalous field dependence of the relaxation rate (approximately $\tau^{-1} \sim \mathrm{H}^{-1}$ at $T=2 \mathrm{~K}$ ) in a wide range of fields between $10^{-2}$ and $3 \mathrm{~T}$ was found, whereas the temperature dependence of $\tau^{-1} \sim T^{\alpha}$ with $\alpha=1.8 \pm 0.2$ holds for fields $H<3 \mathrm{~T}$ and temperatures between 2 and $4 \mathrm{~K}$. The relaxation obwiously goes through some impurities. No satisfactory theoretical explanation of the field dependence seems to exist at present. 